\title{
Controlling the bioreceptor spatial distribution at the nanoscale for single molecule counting in microwell arrays
}

Devin Daems ${ }^{1,{ }^{\dagger}}$, lene Rutten ${ }^{1,{ }^{\dagger}}$, Jonathan Bath ${ }^{2}$, Deborah Decrop ${ }^{1}$, Hans Van Gorp ${ }^{3}$, Elena Pérez Ruiz ${ }^{1}$, Steven De Feyter ${ }^{3}$, Andrew J. Turberfield ${ }^{2}$, Jeroen Lammertyn ${ }^{1, *}$

${ }^{1} \mathrm{KU}$ Leuven, Department of Biosystems, Biosensors group, Willem de Croylaan 42, B-3001 Leuven, Belgium

${ }^{2}$ Oxford University, Department of Physics, Clarendon Laboratory, Parks Road, Oxford, OX1 3PU, United Kingdom

${ }^{3} \mathrm{KU}$ Leuven, Department of Chemistry, Molecular Imaging and Photonics, Celestijnenlaan 200f, B3001 Leuven, Belgium

${ }^{\dagger}$ Contributed equally to this manuscript

*Corresponding author: jeroen.lammertyn@kuleuven.be; +32 16321459.

\section{Abstract}

The ability to detect low concentrations of protein biomarkers is crucial for the early-stage detection of many diseases, and therefore indispensable for improving diagnostic devices for healthcare. Here, we demonstrate that by integrating DNA nanotechnologies like DNA origami and aptamers we can design innovative biosensing concepts for reproducible and sensitive detection of specific targets. DNA origami structures decorated with aptamers were studied as a novel tool to structure the biosensor surface with nanoscale precision in a digital detection bioassay, enabling control of the density, orientation and accessibility of the bioreceptor to optimize the interaction between target and aptamer. DNA origami was used to control the spatial distribution of an in-house generated aptamer on superparamagnetic microparticles, resulting in an origami-linked digital aptamer bioassay to detect the main peanut antigen Ara h1 with 2-fold improved signal to noise ratio and 15-fold improved limit of detection compared to a digital bioassay without DNA origami. Moreover, the sensitivity achieved was 4 orders of magnitude higher than commercially available and literature-reported ELISA techniques. In conclusion, this novel and innovative approach to engineer biosensing interfaces will be of major interest to scientists and clinicians looking for new molecular insights and ultrasensitive detection of a broad range of targets, and, for the next generation of diagnostics. 
For TOC only

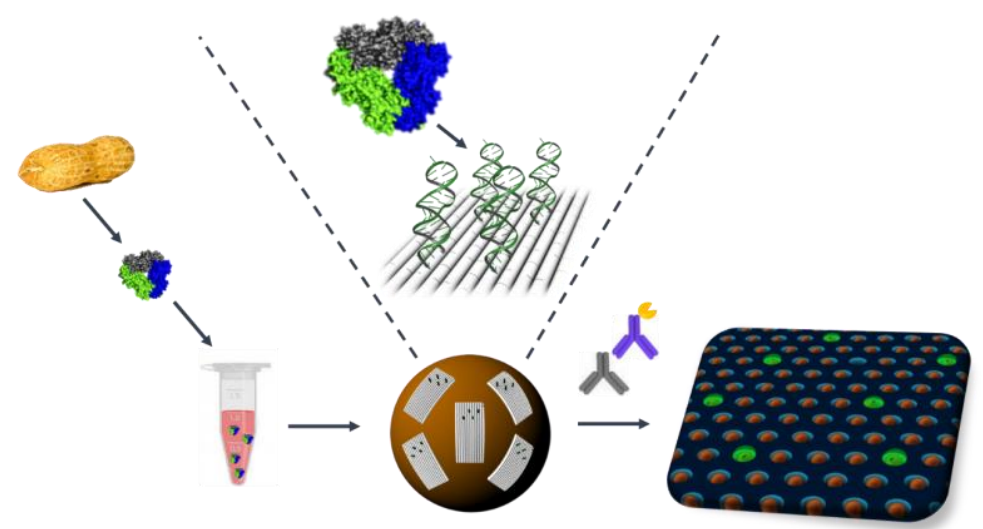

Keywords: Digital bioassay, DNA origami, aptamer, single molecule counting, DNA nanotechnology 
Early-stage detection is crucial for the effective treatment or prevention of many diseases such as cancer, neurological disorders or infectious diseases ${ }^{1-3}$. In this stage of the disease typically only a limited amount of the associated biomarker is present. The low abundance of biomarker in what is typically a limited sample volume makes the ability to detect extremely low concentrations of biomolecules indispensable for improving diagnostics. In addition, in the food industry, the sensitive detection of potential food allergens is essential as in certain cases even trace amounts can induce allergic reactions. Both cases are at the root of an important societal challenge: early-stage detection of disease biomarker and trace amounts of food allergens could significantly improve a patients' quality of life and also reduce the medical and non-medical costs associated with healthcare ${ }^{4}$.

Conventionally, the detection of biomarkers is performed in bulk. However, digital bioassays, such as digital ELISA ${ }^{5-7}$ and digital $\mathrm{PCR}^{8,9}$, have recently emerged in order to meet the desire to improve the sensitivity ${ }^{10,11}$. Digital measurements are performed by subdividing the sample into a large number of smaller volumes in the nano- to picoliter range. This typically results in the presence or absence of a single molecule of analyte within each subvolume according to Poisson statistics ${ }^{5}$. The concept of digitally counting signals generated by isolated target molecules in confined subvolumes makes the digital assay one of the most sensitive techniques available in the biosensing field, capable of detecting biomolecules in extremely low concentrations and down to single molecules.

The bioreceptors used in digital assays are typically antibodies ${ }^{1,7,12}$. In past decades, specific singlestranded DNA or RNA oligonucleotides, called aptamers, have emerged as alternative bioreceptors to antibodies. Aptamers can be selected through a SELEX procedure to bind a target molecule with high affinity and specificity ${ }^{13-16}$. Furthermore, compared to antibodies aptamers do not suffer from batchto-batch variations, increased costs and hampered modification options related to in-vivo production, or decreased stability induced by temperature changes, making them ideal for deployment in biosensing devices ${ }^{17,18}$.

Bioreceptors are traditionally immobilized using covalent attachment, affinity immobilization or selfassembly, resulting in largely uncontrolled and empirical coupling of the bioreceptor to the surface ${ }^{19}$. Steric hindrance and unfavorable orientation can limit the accessibility of the bioreceptor, which leads to a suboptimal interaction with the target molecule. Moreover, areas of the surface not covered by receptors are susceptible to unspecific binding of the sample matrix molecules. All this results in reduced sensitivity, specificity, reproducibility and an overall lack of performance ${ }^{16,20,21}$. For singlemolecule detection concepts such as digital ELISA and digital PCR these issues become even more prominent.

In addition to bioreceptor functions, DNA has been used to design 2D and 3D origami nanostructures. DNA origami ${ }^{22}$ is assembled by hybridizing a multitude of complementary short single-stranded oligonucleotides (called staple strands) to a long single-stranded scaffold to form a variety of multidimensional shapes. Since the introduction of this scaffold-based self-assembling process by Rothemund in 2006, numerous applications of 2D and 3D origami structures have arisen in materials science $^{23,24}$, biomolecular interaction analysis ${ }^{25-28}$, molecular computation ${ }^{29}$ and nanomedicine ${ }^{30-32}$. It has recently been shown that multiple bioreceptors incorporated in a rigid DNA origami structure preserve their ability to bind their target molecules specifically ${ }^{27,33}$ and that the relative spacing of bioreceptors can be used to control their binding affinity ${ }^{34}$. This addressability, together with the ability of creating complex and mechanically robust nanostructures of predefined dimensions ${ }^{35,36}$, makes DNA origami a powerful tool to be used for patterning biosensing surfaces with nanoscale precision for enhancing overall biosensing performance ${ }^{37}$. 
In this work we present for the first time the integration of DNA origami with aptamer nanotechnology to pattern (sub-)micrometer biosensing surfaces with nanoscale precision to develop ultrasensitive digital bioassays. For this purpose, an in-house generated and well-characterized aptamer against peanut allergen Ara $\mathrm{h} 1^{38}$ is used as a model system in a multistep digital bioassay in which the aptamer bioreceptors are precisely distributed and oriented using 2D origami tiles to nanostructure the surfaces of magnetic microparticles.

\section{Results and discussion}

\section{Digital bioassays using aptamers}

Traditionally, antibodies are used as specific bioreceptors to capture the molecule of interest in digital bioassays such as digital ELISA. Inspired by DNA nanotechnology, we explore for the first time the use of a ssDNA aptamer bioreceptor in combination with antibodies to create a single-molecule enzymelinked hybrid aptamer-immunosorbent assay (digital ELHAISA). Our principal innovation is to employ DNA origami for specific attachment of DNA aptamers to superparamagnetic microparticles resulting in optimal accessibility, density and capture efficiency of the bioreceptors to enable digital counting of analyte molecules. In this work the detection of Ara h1, a protein causing an allergenic response to peanuts, is studied as a model system. The detection of Ara h1 is performed in a three-step assay: (1) the protein of interest is captured by a ssDNA aptamer immobilized on the magnetic microparticles; (2) a detection antibody is used to detect the captured protein; and (3) the formed aptamerimmunocomplex is labelled with an enzyme-conjugated secondary antibody (Figure 1).

A

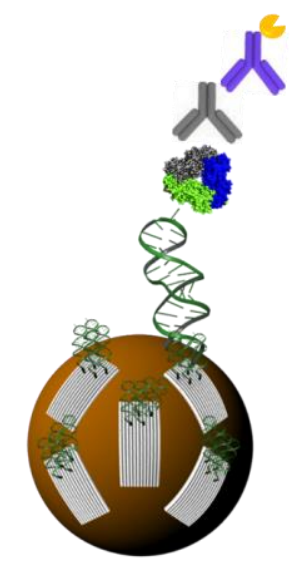

B

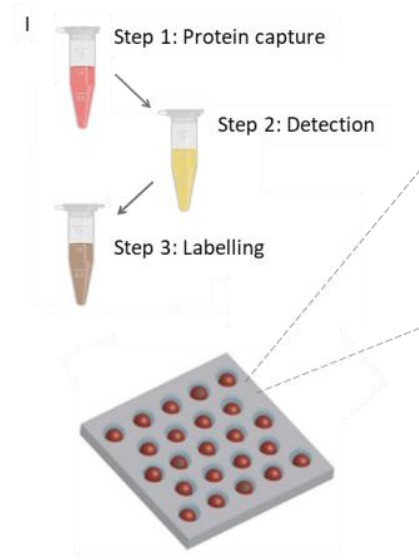

॥

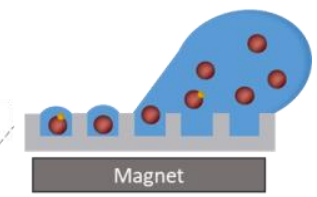

III

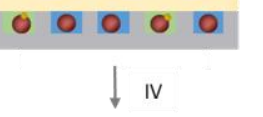

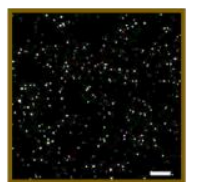

Figure 1: A) Schematic overview of the DNA nanotechnology-based digital capture assay (components not to scale). B) Schematic overview of the assay: I) the immunocomplex is formed in a three-step procedure (i.e. capture of the protein, detection, and final labelling with an enzyme conjugate), II) the magnetic microparticles are loaded into the microwells by moving the sample droplet back and forth over the array in the presence of a magnet, III) the fluorogenic substrate is added and the microwells are subsequently sealed with an oil droplet, forming individual femtoliter droplets of the fluorogenic substrate in the microwells. Magnetic microparticles carrying an aptamer-immunocomplex generate a fluorescent signal. IV) Example of the generated fluorescent image visualized with an inverted fluorescence microscope (scale bar: $100 \mu \mathrm{m}$ ).

In digital assays, the ratio between bound target molecules and magnetic microparticles must be sufficiently small that most particles carry either one target molecule or no target at all. In this limit, determination of the bulk protein concentration can be based on digitally counting the fraction of active particles. If more than $70 \%$ of the magnetic microparticles are active then some are likely to carry more than one enzyme and a digital count of the signal no longer enables the determination of the protein concentration ${ }^{39}$. Moreover, ensuring maximum capture efficiency is of crucial importance. The maximum efficiency of capturing target molecules on aptamer-functionalized magnetic microparticles is greatly dependent of the dissociation constant $\left(\mathrm{K}_{\mathrm{D}}\right)$ of the bioreceptor and the binding kinetics of the aptamer-target complex. 


\section{Directly-coupled aptamer digital bioassay}

As reference system, we coupled a high-affinity in-house selected aptamer against Ara h1 $\left(K_{D}=353\right.$ $\mathrm{nM})^{38}$ as bioreceptor directly to the magnetic microparticles. Using the described immobilization strategy, each magnetic microparticle displays approximately 160,000 aptamers (Supporting Information Figure S1). Assay parameters were optimized in order to maximize the signal-to-noise ratio (SNR) and consequently, to lower the limit of detection (LOD). A secondary enzyme-conjugated antibody was used as a labelling reagent in the digital format to study the relationship between the concentration and the amount of signal generated (Supporting Information Figure S2 and S3). It was shown that the assay is improved by using low concentrations of labelling antibody with an optimal SNR at $130 \mathrm{pM}$ labelling antibody. The optimal ratio of labelling and detection antibody $\left(\mathrm{K}_{\mathrm{D}}=0.52\right.$ $\mathrm{nM}^{40}$ ) was further investigated (Supporting Information Figure S4). A higher concentration of the detection antibody resulted in a higher signal, especially for lower concentrations of Ara h1 target, but also gave higher background signals. Therefore, a combination of $1 \mathrm{nM}$ of detection antibody and 130 pM of labelling antibody was chosen as optimal and used in further experiments.

The sensitivity of the directly-coupled aptamer digital bioassay was determined by establishing a calibration curve based on five concentrations of target Ara h1 in SBT buffer (in the range 0.1 to 1,000 pM) and applying the optimized assay parameters determined above (Figure 2). A sample without Ara h1 was included as negative control. An average seeding efficiency of $\pm 80 \%$ was obtained in the experiments (Supporting Information Figure S5). A 4-parameter logistic model (4PL) was used to describe the experimental data $\left(R^{2}=0.93\right)$. The experimental LOD of the optimally designed bioassay was in the high femtomolar range ( $232 \mathrm{fM}$ ), being close to the theoretically calculated LOD of $150 \mathrm{fM}$ (see Supporting Information). Since the trimeric Ara h1 protein has a molecular weight of $194 \mathrm{kDa}$, the obtained LOD corresponds to $45 \mathrm{pg} / \mathrm{mL}$ Ara h1. The sensitivity reported in literature is $12 \mathrm{ng} / \mathrm{mL}$ for an ELISA format ${ }^{41}$ and $450 \mathrm{ng} / \mathrm{mL}$ for the lateral flow assay ${ }^{42}$. Therefore, it can be concluded that a $1,000 \times$ enhancement of LOD compared to values previously reported was achieved. These results also show that, even using an aptamer with relatively high $\mathrm{K}_{\mathrm{D}}$ and low capturing efficiencies, it is still possible to detect high $\mathrm{fM}$ analyte concentrations. Based on our results it can be concluded that the directlycoupled aptamer digital bioassay is probably at its limit of sensitivity and that for achieving an even better LOD (down to low $\mathrm{fM}$ range) most probably an aptamer with lower $\mathrm{K}_{\mathrm{D}}$ or improved adsorption kinetics (through enhanced accessibility of the aptamers) is needed.

A

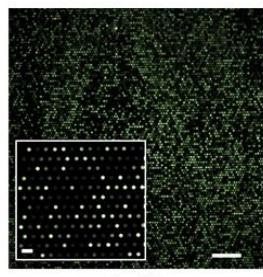

$1 \mathrm{nM}$

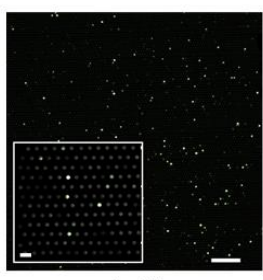

$1 \mathrm{pM}$

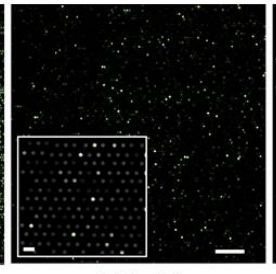

$100 \mathrm{pM}$

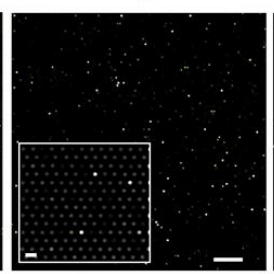

$100 \mathrm{fM}$

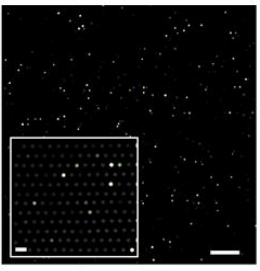

$10 \mathrm{pM}$

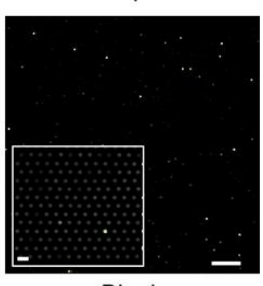

B

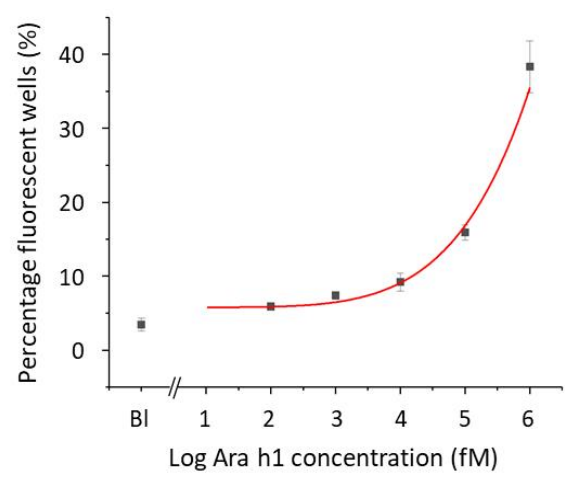

Figure 2: Peanut allergen bioassay on the digital platform. A) Representative fluorescent images of the digital quantification of Ara h1 (1,000 pM to 0.1 pM and Blank) with a directly-coupled aptamer digital bioassay (scale bar represents $100 \mu \mathrm{m}$ ). Insert: Detailed view of the array showing an overlay of the fluorescent signal and corresponding brightfield image of the 
magnetic particles, seeded in the microwells. Each well represents one of the 3 following situations: (1) empty well, (2) well with bead in absence of target (i.e. no fluorescent signal) and (3) well with bead in presence of target (i.e. fluorescent signal) (scale bars represent $10 \mu \mathrm{m})$. B) Calibration curve for Ara h1 representing the percentage of fluorescent wells for each concentration. Error bars represent one standard deviation $(n=3)$.

\section{Design, development and validation of DNA origami structures}

The aim of this work is to apply DNA nanotechnology to increase the sensitivity of the directly-coupled aptamer digital bioassay by enhancing the accessibility of the bioreceptor coupled to the biosensing surface, thus promoting improved biomolecular interaction between bioreceptor and target. We integrate for the first time DNA origami with aptamer technology to pattern (sub-) micrometer 3D biosensing surfaces with nanoscale precision to develop ultrasensitive origami-linked aptamer digital bioassays. A rectangular DNA origami structure was designed and developed (Supporting Information Figure S6). The 2D origami core ${ }^{43}$ was modified to allow specific attachment of the DNA origami structures to (i) the magnetic microparticles $(\varnothing 2.7 \mu \mathrm{m})$ and (ii) the anti-Ara h1 aptamers by specific hybridization with complementary ssDNA staple extensions. These two sets of ssDNA staple extensions were positioned on opposite sides of the DNA origami core leading to 8 elongated staples on the bottom surface and 4 on the top surface for attachment to the magnetic microparticle and bioreceptors respectively (Figure 3 ).

A

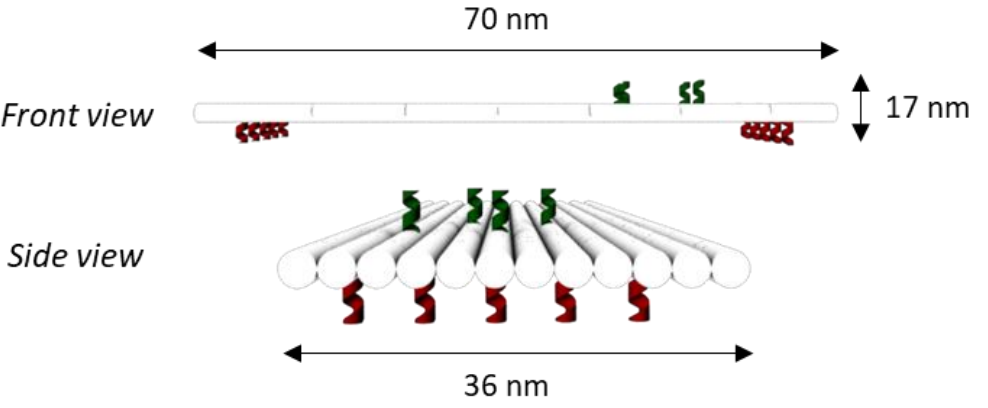

B

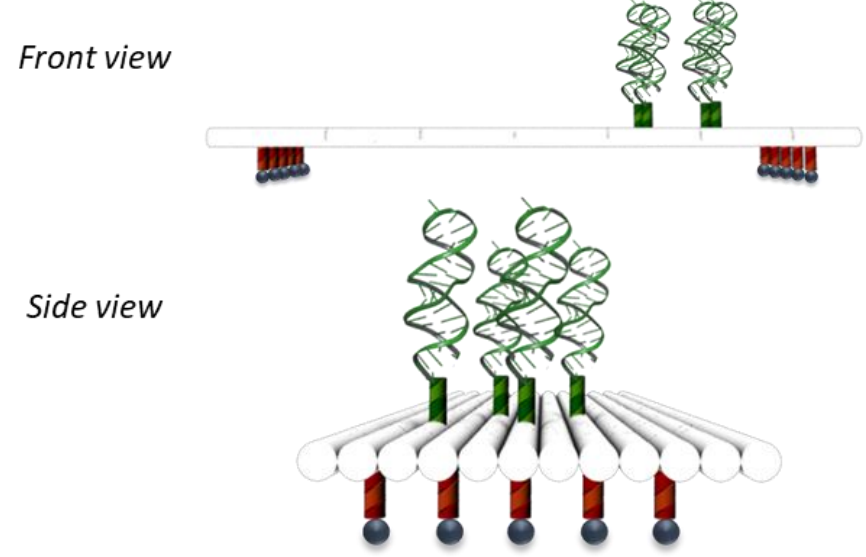

C

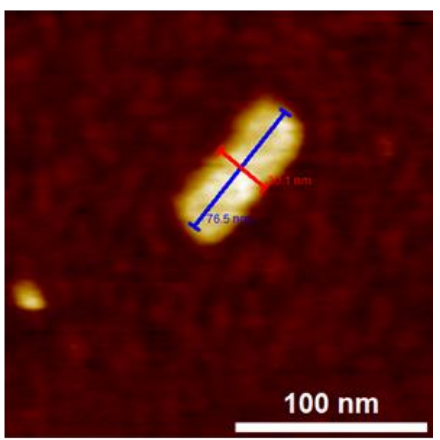

Figure 3: 2D DNA origami design and characterization. A) Schematic representation of the DNA origami structures with ssDNA extensions for bioreceptor coupling (aptamer set, green) and for surface attachment (microparticle set, red). B) Schematic illustrations of the front and side view, representing the 2D DNA origami with addition of both ssDNA sets, aptamer bioreceptors (green) and biotin-modified ssDNA strands (red). C) AFM image of the 2D origami (scale bar represents $100 \mathrm{~nm}$ ).

In order to evaluate the robustness of the DNA origami structures for reproducible biosensing experiments, we first examined their stability under different buffer conditions using gel electrophoresis. The stability of the DNA origami structures was demonstrated over a $\mathrm{pH}$ range (between 7 and 9) typical for biosensing and by incubating the structures for more than 30 min over a broad temperature range (between 20 and $60{ }^{\circ} \mathrm{C}$ ) (Supporting Information Figure S7). As shown in Figure S7B and C, denaturation of the different DNA origami structures starts to occur at temperatures 
close to $60{ }^{\circ} \mathrm{C}$. A melting temperature of $56.5^{\circ} \mathrm{C}$ was confirmed with high resolution melting (HRM). Reduced melting temperatures may be an indication of destabilizing factors or suboptimal design ${ }^{44}$. The range of $\mathrm{pH}$ values tested had no impact on the stability of the DNA origami structures (Figure S7A). In addition, the origami was imaged using atomic force microscopy (AFM) (Figure 3 and Supporting Information Figure S8). The AFM images clearly show that the experimentally determined dimensions of the DNA origami structures ( $L: 73.1 \pm 3.1 \mathrm{~nm}$ and $\mathrm{W}: 29.5 \pm 0.8 \mathrm{~nm}, \mathrm{n}=10$ ) match well the theoretical size calculations ( $\mathrm{L}: 70 \mathrm{~nm}, \mathrm{~W}: 36 \mathrm{~nm}$ ).

\section{Attachment of functionalized origami structures to magnetic microparticles}

To test whether the developed DNA origami is suited for patterning the magnetic particles with aptamers, we first studied the accessibility of the complementary ssDNA staple extensions by means of gel electrophoresis experiments. We hybridized biotinylated ssDNA oligonucleotides to the ssDNA microparticle set, fluorescein amidite (FAM) labeled oligonucleotides to the ssDNA aptamer set, and/or both types of oligonucleotides to both ssDNA sets on the same DNA origami. The origami contained one Cy5 labelled staple strand for visualization. Cy3-labelled streptavidin was added to modified origami structures functionalized with biotinylated ssDNA oligonucleotides in order to evaluate the coupling of the biotinylated ssDNA to streptavidin (as a proxy for the streptavidin-coated magnetic particles). The images in Figure 4A clearly indicate the accessibility of both the ssDNA sets for hybridization with complementary oligonucleotides and of the biotinylated oligonucleotides for binding to streptavidin.

A

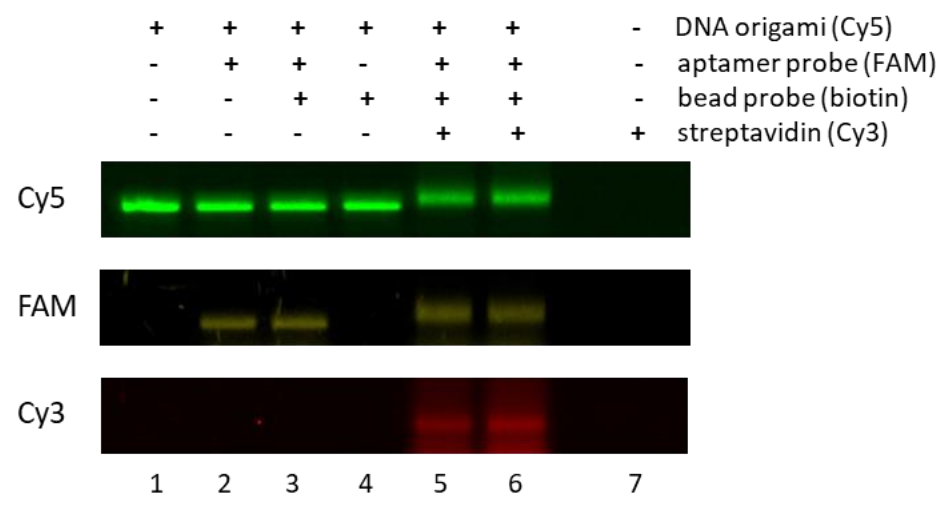

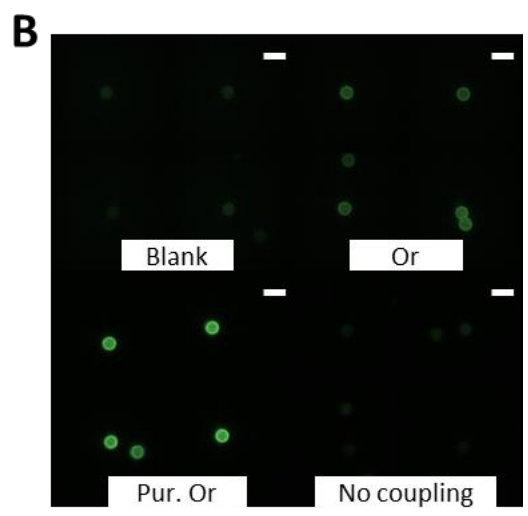

Figure 4: A) Characterization of the origami structure with gel electrophoresis using $0.7 \%$ agarose gel: Lanes 1 to 6 contain Cy5-labelled DNA origami. The accessibility of the aptamer attachment sites was tested by addition of a complementary FAMlabelled DNA strand. The accessibility of the magnetic microparticle attachment site was tested by addition of a complementary biotinylated strand and Cy3-labelled streptavidin (lane 5 added together, lane 6 added sequentially). The signal in the FAM and Cy3 channels demonstrates accessibility of both aptamer and microparticle attachment sites. B) Fluorescent images of: empty magnetic microparticles (Blank), Cy5 labelled origami coupled to magnetic microparticles (Or), purified Cy5 labelled origami coupled to magnetic microparticles (Pur. Or) and Cy5 labelled origami without biotin handles coupled to magnetic microparticles (no coupling). Scale bars represent $5 \mu \mathrm{m}$.

Streptavidin-coated magnetic microparticles covered with fluorescently labeled DNA origami were imaged by epifluorescence microscopy (Figure 4B and Supporting Information S9). Fluorescence micrographs clearly show the presence of the fluorescently labeled origami, proving that the DNA origami structures could be successfully immobilized on the surface of the magnetic microparticles. Furthermore, PEG purification of the DNA origami structures resulted in the desired removal of excess staple strands and ssDNA sets ${ }^{45}$. This resulted in an enhanced fluorescent signal, indicating a higher coupling yield of the DNA origami to the surface of the magnetic microparticle. 
In summary, we have designed and characterized DNA origami structures that incorporate coupling groups suitable for further use in biosensing applications. We have demonstrated the attachment of these origami structures to streptavidin-coated magnetic microparticles, used in digital assays.

\section{Origami-linked aptamer digital bioassay}

We studied the impact of coating the magnetic microparticles with 2D DNA origami structures on the performance of the digital bioassay. Working with DNA origami allowed us to control the surface density of aptamers by varying the number and spacing of the aptamers per DNA origami structure. Using this origami-based immobilization strategy, on average 7,500 origami structures (containing each a maximum of 4 aptamers) were coupled to each magnetic microparticle, at least five times fewer bioreceptors per microparticle than in the directly-linked digital aptamer bioassay (Supporting Information Figure S10). Figure 5A shows the biosensor signal for 4 different DNA origami-aptamer spatial configurations (Supporting Information Figure S11) and the blank in the presence of $10 \mathrm{pM}$ of analyte Ara h1. The blank, lacking the presence of the target specific aptamer bioreceptors on the DNA origami nanostructure surface, demonstrates no significant non-specific interaction between the Ara h1 protein and the DNA origami structures immobilized on the microparticle surface.

Although far fewer aptamers were present on the surface, the configuration with 1 aptamer per origami gave a similar signal ( $10 \%$ active wells and a CV of $5 \%$ ) to the directly coupled aptamers (9\% active wells and a CV of $13 \%$ ). The improved reproducibility is very likely due to a more consistent upwards orientation and reduced steric hindrance of the aptamers when coupled to an origami adapter rather than directly to the microparticle surface ${ }^{34}$. Moreover, the observation that $10 \%$ of beads are active using 1 aptamer per origami implies that the availability for binding of each origamibound aptamer is close to that in free solution: in this limit the average number of targets bound per magnetic microparticle would be approximately equal to the number of aptamers per magnetic microparticle $\times$ target concentration $/ K_{D}=7,500 \times 0.010 n M / 353 n M=0.21$. Directly coupled aptamers, on the other hand, are much less available.

Adding a second aptamer spaced $6.2 \mathrm{~nm}$ from the first one, and a third in a triangular configuration with inter-aptamer distances of $6 \mathrm{~nm}$ and $6.2 \mathrm{~nm}$ (Supporting Information Figure S11), did not significantly increase the detected signal (10\% active wells / CV of $2.5 \%$ and $11 \%$ active wells / CV of $2.1 \%$ respectively). We infer that these aptamer spacings are suboptimal for multiple aptamer-target interactions. However, adding a $4^{\text {th }}$ aptamer to the DNA origami, increasing the maximal distance between 2 aptamers to $10.5 \mathrm{~nm}$ (Supporting Information Figure S11), gave a strongly enhanced signal ( $21 \%$ active wells / CV of $4.8 \%$ ) compared to the reference system and the other configurations. Most probably this can be attributed to polyvalent interactions ( 2 aptamers binding to 1 target) and hence a stronger interaction and higher apparent affinity for the Ara h1, a trimeric protein with a radius of approximately $9 \mathrm{~nm}^{46,47}$. Generalizing, this method could be used for other ligands with repeating structures. Moreover, different receptors (aptamers and/or antibodies) targeting different regions of the same ligand could be combined in a similar way for a non-repeating structure by positioning them on the DNA origami with nanoscale precision.

In summary, for the structures with 1, 2 and 3 aptamers we observed signals comparable to the reference with a five-fold greater surface density of directly coupled aptamers. A 2.3-fold increase in signal was measured for the DNA origami with 4 aptamers (Figure 5A and Supporting Information Figure S11). We attribute the enhanced signal to a more favorable positioning of the origami-coupled aptamers relative to directly-coupled aptamers and (in the case of four aptamers per origami) to an increase in avidity due to polyvalent binding enabled by the DNA origami allowing a nanoscale precise positioning of the bioreceptors. 

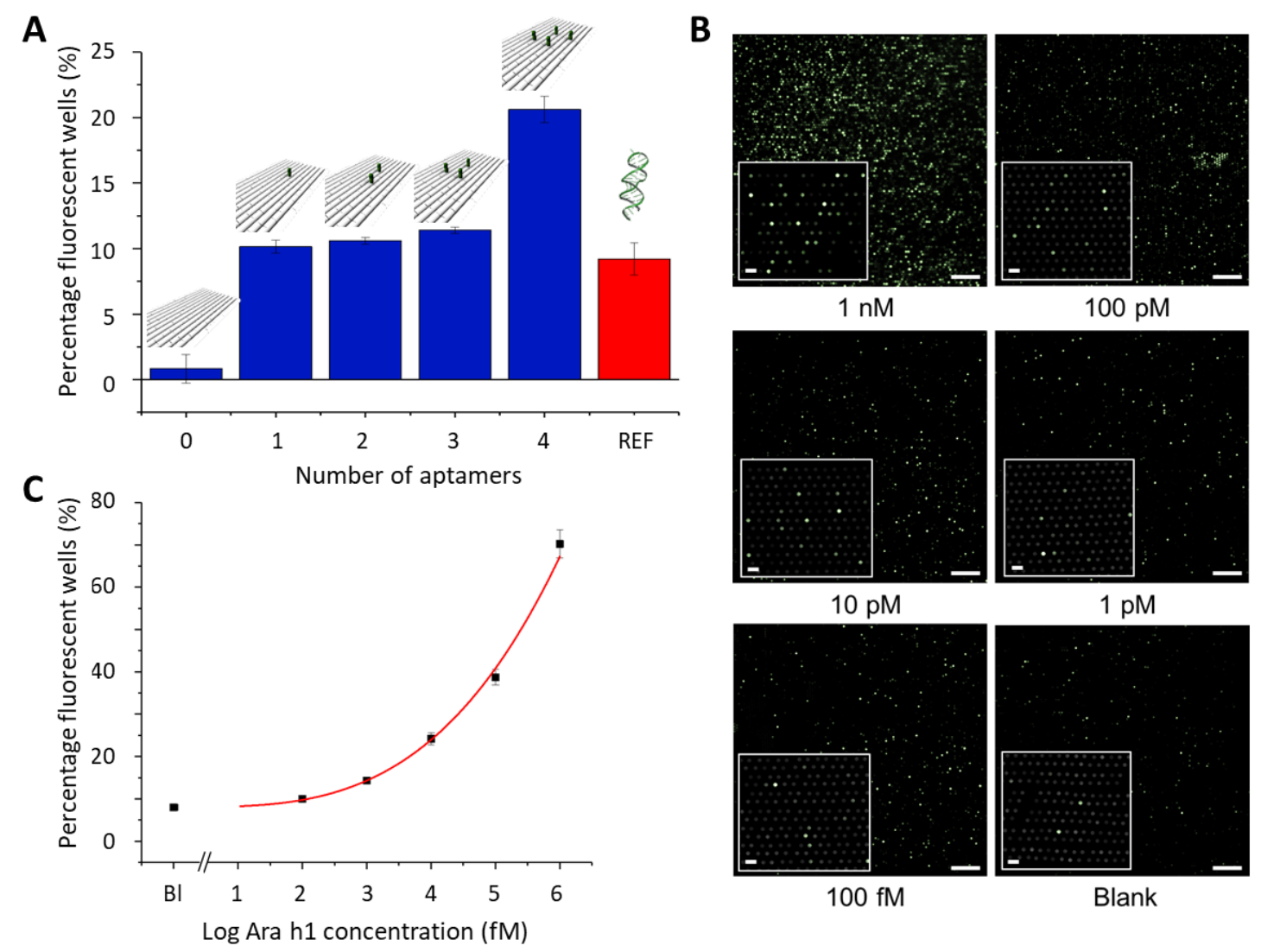

$1 \mathrm{nM}$

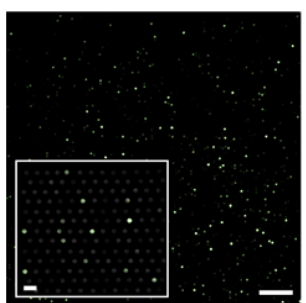

$100 \mathrm{pM}$

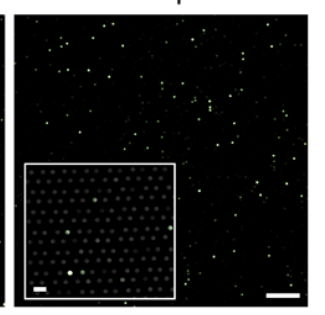

$10 \mathrm{pM}$

$1 \mathrm{pM}$

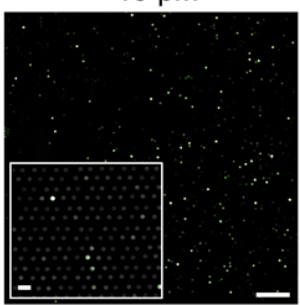

$100 \mathrm{fM}$

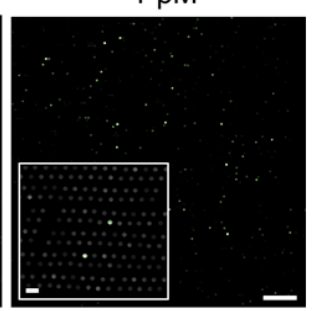

Blank

Figure 5: Peanut allergen bioassay on the digital platform using DNA origami to control the patterning of streptavidin coated magnetic microparticles with aptamers for peanut allergen Ara h1. A) Experimental results of a digital bioassay to detect 10 pM Ara h1 with different numbers of aptamers coupled to each DNA origami structure; the reference system has a fivefold higher surface density of aptamers coupled directly to the microparticles. B) Representative fluorescent images of the digital quantification of Ara h1 (from 1,000 pM to 0.1 pM and Blank) with the DNA nanotechnology-based digital assay (scale bar represents $100 \mu \mathrm{m})$. Insert: Detailed view of the array showing an overlay of the fluorescent signal and corresponding brightfield image of the magnetic particles, seeded in the microwells. Each well represents one of the 3 following situations: (1) empty well, (2) well with bead in absence of target (i.e. no fluorescent signal) and (3) well with bead in presence of target (i.e. fluorescent signal) (scale bars represent $10 \mu \mathrm{m}$ ). C) Calibration curve for Ara h1 using 4 aptamers coupled to one DNA origami. Error bars represent one standard deviation $(n=3)$.

In Figure $5 C$, a calibration curve $\left(R^{2}=0.99\right)$ is presented for quantification of the aforementioned Ara h1 concentrations ( $0.1 \mathrm{pM}$ to $1,000 \mathrm{pM}$ and a blank) using 4 aptamers coupled to one DNA origami: corresponding micrographs are shown in Figure 5B and Supporting Information Figure S12. The negative control, not containing any Ara h1 protein did not result in a significant percentage of fluorescent wells. This result shows there is limited non-specific interaction between the DNA origami and the assay components. In addition, the specificity of the assay was challenged against two other biomarkers based on which no significant non-specific interactions were reported (Figure S14). By using DNA origami to nanostructure the biosensing interface, we were able to lower the LOD to $18 \mathrm{fM}$ $(3.5 \mathrm{pg} / \mathrm{mL}), 15$-fold lower than that for the corresponding directly-linked digital aptamer bioassay without DNA origami. Moreover, due to the decrease in signal variation from $11 \%$ (without origami) to $4 \%$ (with origami), the average SNR was enhanced from 13.5 to 24.0 for the digital assay without origami compared to with origami. The dynamic range covers detection of Ara h1 protein from $18 \mathrm{fM}$ to $1 \mathrm{nM}$ when using origami-linked aptamers and from $232 \mathrm{fM}$ to $6.6 \mathrm{nM}$ for the directly-coupled aptamers, both addressing a significant range of concentrations and achieving a one order of magnitude increase when using origami-linked aptamers. The time-to result amounts to $3 \mathrm{~h} 30 \mathrm{~min}$, leaving room for improvement as this was not optimized towards the shortest possible detection time. In theory, taking into account the diffusion limitations ${ }^{48}$ the incubation times could be reduced with 
$60 \%$ without significantly altering the assay performance. Despite the lower density of bioreceptors on the biosensing surface, nanostructuring magnetic microparticles with DNA origami led to the development of a biosensor with enhanced sensitivity, reproducibility, SNR and dynamic range compared to biosensors with directly-attached aptamers. Although further optimization is still possible, the new strategy of using DNA nanotechnology for digital bioassay already indicates an improved sensitivity towards functional Ara h1 compared to the high pM range of previously reported peanut allergen assays ${ }^{40,41}$. Although the included control experiments provide promising results showing limited non-specific interactions of both the Ara h1 molecules and the assay components with and without DNA origami nanostructures, it should be noted that starting from real samples might result in slightly higher LOD values (10-fold) due to the increased complexity of the detection matrix. However, by using magnetic microparticles we can include several pretreatment and washing steps in the assay to limit or even avoid non-specific signals due to biological matrices ${ }^{49}$. A more in depth assessment of the robustness of DNA origami structures in biological matrices would exceed the focus of the research presented in this paper.

\section{Conclusions}

In conclusion, our results highlight the potential for DNA nanotechnology to improve digital bioassays. We implemented for the first time a DNA aptamer as bioreceptor in a digital bioassay, outperforming the sensitivities previously reported in literature. To further improve the sensitivity of the digital aptamer bioassay, a 2D origami structure was designed, developed and characterized to attach DNA aptamers to the biosensing surface with precise control of local environment and predictable orientation. The effect of the DNA origami was to enhance interactions between aptamer and target, leading to increased reproducibility, SNR and sensitivity. Therefore, both DNA nanotechnologies (aptamers and DNA origami) are recognized as promising building blocks in digital bioassay development. Further research will focus on a DNA-only digital bioassay, including DNA-based signal generation and amplification. Our DNA-based digital assay concept will also be integrated with an inhouse developed digital microfluidic platform for ultrasensitive, compact, cheap and fast detection of target molecules.

\section{Material and Methods}

\section{Reagents}

All chemicals were of analytical reagent grade and purchased from The Merck group - Sigma Aldrich (Diegem, Belgium), unless stated otherwise. All buffer solutions were prepared using deionized water filtered by a Milli-Q Plus system (The Merck group - Millipore, Marlborough, MA, USA). Streptavidincoated superparamagnetic microparticles (LodeStars, $\varnothing 2.7 \mu \mathrm{m}$ ) were obtained from Agilent (Santa Clara, CA, USA). The monoclonal antibody against Ara h1 and the purified natural Ara h1 allergen were purchased from Indoor Biotechnologies Limited (IBL, Cardiff, UK). The $\beta$-galactosidase-coupled labelling antibody was purchased from Abcam (Cambridge, UK). The Human $\alpha$-thrombin was purchased from Haematologic Technologies (Essex, VT, USA). The immunoglobulin E (IgE), Human Myeloma Plasma, Kappa, was purchased from Athens Research \& Technology (Athens, GA, USA). The fluorogenic substrate, fluorescein di- $\beta$-D-galactopyranoside (FDG), originated from Sigma-Aldrich (St. Louis, MO, USA) and the plus one cover fluid (silicon oil) from GE Healthcare Life Sciences (Little Chalfont, UK). All unmodified, biotin-modified and fluorescent labelled oligonucleotides were purchased from Integrated DNA Technologies (IDT, Haasrode, Belgium) as desalted products and delivered lyophilized in 96-well plates or vials (Supporting Information Table S1 and S2). 


\section{Microwell-array microfabrication}

The microwell arrays were fabricated using a dry "lift-off" technique earlier described by our group ${ }^{48}$. In short, glass wafers ( $1 \mathrm{~mm}$ thickness) were coated with Teflon-AF${ }^{\circledR}(3 \mu \mathrm{m})$, after first coating a thin layer of fluoroalkylsilane to improve its adhesion. Then, a Parylene-C shadow mask and an aluminum hard mask were applied on top of the Teflon-AF layer. Using standard photolithographic processes and aluminum etchant, the microwell array was subsequently transferred into the aluminum hard mask. The exposed underlying Parylene- $\mathrm{C}$ and Teflon-AF layers were etched using $\mathrm{O}_{2}$-plasma, revealing the hydrophilic glass surfaces. Finally, the Parylene-C shadow mask and aluminum hard mask were peeled off using tweezers. The resulting hydrophobic glass chip contained 62,500 hydrophilic-in-hydrophobic (HIH) microwells with a diameter of $4.5 \mu \mathrm{m}$, a depth of $3 \mu \mathrm{m}$ and a volume of $38 \mathrm{fL}$. The wells occur at a center-to-center distance of $8 \mu \mathrm{m}$ and were organized in square shaped patches of $2 \times 2 \mathrm{~mm}^{2}$.

\section{Functionalization of magnetic microparticles with anti- Ara h1 aptamer}

Streptavidin-coated paramagnetic microparticles were functionalized with biotinylated anti-Ara h1 aptamers. First, $3 \mu \mathrm{L}$ of the magnetic microparticles were mixed with $100 \mu \mathrm{L}$ of SuperBlock buffer (SB, Thermo Scientific, Waltham, MA, USA) to obtain a concentration of $2.4 \times 10^{6}$ magnetic microparticles per $100 \mu \mathrm{L}$. Second, the magnetic microparticle solution was washed twice with $100 \mu \mathrm{L}$ of SB. Afterwards a mixture of magnetic microparticles $(10 \mu \mathrm{L})$ and biotinylated aptamer $\left(20 \mu \mathrm{L}, 2.5 \times 10^{-8} \mathrm{M}\right.$ in $1 \times \operatorname{TEMg}(10)\left(5 \mathrm{mM}\right.$ Tris base, $1 \mathrm{mM} \mathrm{Na}{ }_{2}$ EDTA and $\left.10 \mathrm{mM} \mathrm{MgCl}_{2}, \mathrm{pH} 7.6\right)$ with $200 \mathrm{mM} \mathrm{NaCl}$ ) were incubated at room temperature for $45 \mathrm{~min}$ in $1 \times \mathrm{TEMg}(10)$ with $200 \mathrm{mM} \mathrm{NaCl}(70 \mu \mathrm{L})$ while slowly rotating. After functionalization, the magnetic microparticles were washed 6 times with $1 \times \operatorname{TEMg}(10)$ with $200 \mathrm{mM} \mathrm{NaCl}$ and stored in this buffer at $4{ }^{\circ} \mathrm{C}$ until further use.

\section{Capturing, detection and labelling of target: directly-linked digital aptamer bioassay}

The assay started with the dilution of the stock solution of purified Ara h1 protein ( $50 \mathrm{nM})$ to the desired concentrations in TGK buffer ( $25 \mathrm{mM}$ Tris base, $192 \mathrm{mM}$ Glycine, $5 \mathrm{mM} \mathrm{K}_{2} \mathrm{HPO}_{4}, 0.1 \%$ Tween20 and $0.15 \% \mathrm{w} / \mathrm{v} \mathrm{BSA}, \mathrm{pH}$ 8.3). Next, the magnetic microparticles functionalised with aptamers were washed twice with TGK buffer. Afterwards, the TGK buffer was removed and $100 \mu \mathrm{L}$ of the prepared protein solutions were added to the magnetic microparticles. These mixtures were incubated for 120 min while slowly rotating at room temperature. After incubation, the magnetic microparticles were washed twice in PBST (Phosphate buffered Saline with $0.1 \%$ Tween20, pH 7.4). Subsequently $100 \mu \mathrm{L}$ of the detection antibody diluted in SBT (SB with $0.05 \%$ Tween 20) was added and this combination was incubated for $30 \mathrm{~min}$ while slowly rotating at room temperature. Afterwards the samples were washed two times in PBST. The samples were then incubated in $100 \mu \mathrm{L}$ of SBT buffer with labelling antibody, for $30 \mathrm{~min}$ at room temperature. As presented in Supporting Information Table S3, different concentrations of both the detection and labelling antibodies were tested with the purpose of optimizing the assay. Finally, the particles were washed 6 times in PBST before being resuspended in $10 \mu \mathrm{L}$ of SBT.

\section{Seeding single magnetic microparticles}

After target capturing and labelling, the magnetic microparticles were seeded with single particle resolution in the microwell array containing $62,500 \mathrm{HIH}$ wells that was fabricated following the aforementioned protocol. To do this, a $10 \mu \mathrm{L}$ droplet, containing the suspended magnetic microparticles, was shuttled back and forth over the array with a micropipette while a magnet (NdFeB, $6 \mathrm{~mm}$ diameter, $12.7 \mathrm{~N}$, Supermagnete, Gottmadingen, Germany) was placed under the array to attract the magnetic microparticles into the microwells. The droplet was moved over the array surface for at least 10 cycles in order to obtain a high seeding efficiency ( $\geq 80 \%$ ) (Figure S13). Subsequently, a $30 \mu \mathrm{L}$ droplet containing $100 \mu \mathrm{M}$ solution of the fluorogenic substrate FDG in PBS (Phosphate Buffered Saline, pH 7.4) was placed over the array and a $180 \mu \mathrm{L}$ droplet of oil was placed on top. Finally, the 
droplet of FDG substrate was carefully pipetted away from the array, thereby leaving femtoliter-sized droplets of FDG inside every microwell, while the microwells were sealed with the oil, thus preventing evaporation.

\section{Data acquisition and analysis}

After particle seeding in the microwell array, the plate was imaged on an inverted fluorescence microscope (Olympus IX71, Olympus Corporation, Tokyo, Japan) equipped with a $20 \times$ objective and a EM-CCD Digital Camera (Hamamatsu Photonics, Hamamatsu, Japan). A background image was first taken at $0 \mathrm{~min}$ and subtracted from a second image taken after $20 \mathrm{~min}$ of incubation with the substrate. A WIBA filter (excitation filter BP460-495, emission filter BA510-550 and dichromatic filter BM505) was used to select the correct excitation and emission wavelengths and neutral density filters were applied to decrease the light intensity to $3 \%$. Finally, a third bright field image was taken to visualize the total number of seeded particles. This was done by exposing the particles to high intensity light using a WIGA filter (excitation filter BP530-550, emission filter BA575-625 and dichromatic filter BM570) to visualize their autofluorescence. All the images were acquired with an exposure time of $1 \mathrm{~s}$ and a gain of 1 using the Hokawo software from Hamamatsu Photonics. The ratio of active magnetic microparticles was determined by dividing the number of fluorescent wells (NFW) by the total number of seeded magnetic microparticles (NP). NFW and NP were obtained by processing the images with ImageJ software (US National Institutes of Health, USA), counting the number of NFW in the background-subtracted images. Although slight differences in intensity between different fluorescent microwells could be noticed, this was not taken into account during the well-count as these differences, in our experimental conditions, do not represent multiple target bindings but are merely the result of enzyme heterogeneity and the pre-coupling of multiple enzymes per labelling antibody.

\section{DNA origami}

A rectangular 2D DNA origami structure was designed using caDNAno (caDNAno 2.2.0, MIT License, USA $)^{50}$. The complete list of oligonucleotide sequences used for folding pKD1 plasmid-derived singlestranded DNA scaffold ${ }^{43}$ into the desired shapes is shown in Supporting Information Table S2. This DNA origami structure has been designed such that one side can couple to the magnetic particles and the opposite side can hybridize to a bioreceptor. DNA origami with attached bioreceptors and biotinmodified ssDNA strands at predefined positions was assembled by cooling the scaffold ( $100 \mathrm{nM}$ unless stated otherwise) with a 2-fold excess of staples from $96{ }^{\circ} \mathrm{C}$ to $25{ }^{\circ} \mathrm{C}$ (ramp speed: $-1{ }^{\circ} \mathrm{C} / \mathrm{min}$ ) in $1 \times$ TEMg(10) with $200 \mathrm{mM} \mathrm{NaCl}$. Excess staples were removed using a PEG purification method ${ }^{45}$.

\section{AFM imaging}

AFM images where obtained by depositing $5 \mu \mathrm{L}$ of the DNA origami solution onto a piece of freshly cleaved mica, Grade V4 (Structure Probe Inc., West Chester, PA, USA). The droplet was left unperturbed for $1 \mathrm{~min}$ to promote DNA adsorption before washing the substrate with $20 \mathrm{~mL}$ of MQ water and dried under a gentle stream of argon. The samples where imaged using a Cypher ES system (Oxford Instruments Asylum Research Inc., Santa Barbara, CA, USA) using OMCL-AC240TS probes (Olympus Corporation, Tokyo, Japan). Subsequent image analysis was performed using SPIP 6.3.5 software (Image Metrology, Hørsholm, Denmark).

\section{Gel electrophoresis}

Unless stated otherwise, 2D DNA origami structures were run on a $0.7 \%$ agarose gel using a loading dye (50\% glycerol, $50 \% 1 \times$ TE buffer and traces of bromophenolblue) with $1 \times$ TAE (40 mM Trisacetate, 1 mM EDTA (50 x Fisher Bioreagents, Hampton, NH, USA), pH 8.3) for $1 \mathrm{~h}$ (at $100 \mathrm{~V}$ ) and stained with SyBrGold (20 min). Band intensities were visualized using a Pharos FX ${ }^{\mathrm{TM}}$ Plus Molecular Imager (Bio-rad, Hercules, CA, USA). 


\section{Imaging of functionalized magnetic microparticles}

Imaging was performed, both in bright-field and fluorescence modes, using a Cy5 dye-matching laser line. Functionalized magnetic microparticles were inspected using a $100 \times$ NA 1.45 oil immersion objective on an inverted Nikon Eclipse TE2000U microscope equipped with a mercury arc lamp, a commercial fluorescence filter set and a charge-coupled device digital camera (Thorlabs 340M-GE, Newton, NJ, USA). All measurements were carried out in EPI fluorescence mode.

\section{Acknowledgements}

We gratefully acknowledge financial support from KU Leuven (IOF/KP/12/002-Nanodiag; PDM/15/111 Devin Daems, OT 13/058), and Fund for Scientific Research (FWO G086114N, FWO-Flanders Postdoctoral Fellow Devin Daems 12U1618N, FWO-Flanders Research Stay Devin Daems V419416N, FWO-Flanders Doctoral grant lene Rutten 1 S30016N). AJT acknowledges a Royal Society Wolfson Research Merit Award.

\section{Supporting Information}

The Supporting Information is available free of charge on the ACS Publications website (se-2019008778_SI.pdf). The Supporting Information contains: sequences of oligonucleotides used in this work; determination of the number of aptamers per magnetic microparticles; optimization of experimental conditions; seeding efficiency for digital ELISA using aptamers and DNA origami; theoretical calculation LOD; characterization and validation of the developed DNA origami structure; schematic illustration of the aptamer positions on the origami structures and corresponding micrographs; assay selectivity towards other biomarkers.

\section{References}

(1) Leirs, K.; Tewari Kumar, P.; Decrop, D.; Pérez-Ruiz, E.; Leblebici, P.; Van Kelst, B.; Compernolle, G.; Meeuws, H.; Van Wesenbeeck, L.; Lagatie, O.; et al. Bioassay Development for Ultrasensitive Detection of Influenza A Nucleoprotein Using Digital ELISA. Anal. Chem. 2016, $88,8450-8458$.

(2) Azimzadeh, M.; Nasirizadeh, N.; Rahaie, M.; Naderi-Manesh, H. Early Detection of Alzheimer's Disease Using a Biosensor Based on Electrochemically-Reduced Graphene Oxide and Gold Nanowires for the Quantification of Serum microRNA-137. RSC Adv. 2017, 7, 55709-55719.

(3) Afsahi, S.; Lerner, M. B.; Goldstein, J. M.; Lee, J.; Tang, X.; Bagarozzi, D. A.; Pan, D.; Locascio, L.; Walker, A.; Barron, F.; et al. Novel Graphene-Based Biosensor for Early Detection of Zika Virus Infection. Biosens. Bioelectron. 2018, 100, 85-88.

(4) Abbott, A. A Problem for Our Age. Nature 2011, 475, S2-S4.

(5) Rissin, D. M.; Walt, D. R. Digital Concentration Readout of Single Enzyme Molecules Using Femtoliter Arrays and Poisson Statistics. Nano Lett. 2006, 6 (3), 520-523.

(6) Rissin, D. M.; Kan, C. W.; Campbell, T. G.; Howes, S. C.; Fournier, D. R.; Song, L.; Piech, T.; Patel, P. P.; Chang, L.; Rivnak, A. J.; et al. Single-Molecule Enzyme-Linked Immunosorbent Assay Detects Serum Proteins at Subfemtomolar Concentrations. Nat. Biotechnol. 2010, 28 (6), 595-599.

(7) Witters, D.; Knez, K.; Ceyssens, F.; Puers, R.; Lammertyn, J. Digital Microfluidics-Enabled Single-Molecule Detection by Printing and Sealing Single Magnetic Beads in Femtoliter Droplets. Lab Chip 2013, 13 (11), 2047-2054. 
(8) Vogelstein, B.; Kinzler, K. W. Digital PCR. Proc. Natl. Acad. Sci. U. S. A. 1999, 96, 9236-9241.

(9) Baker, M. Digital PCR Hits Its Stride. Nat. Methods 2012, 9 (6), 541-544.

(10) Kühnemund, M.; Witters, D.; Nilsson, M.; Lammertyn, J. Circle-to-Circle Amplification on a Digital Microfluidic Chip for Amplified Single Molecule Detection. Lab Chip 2014, 2983-2992.

(11) Kelley, S. O.; Mirkin, C. A.; Walt, D. R.; Ismagilov, R. F.; Toner, M.; Sargent, E. H. Advancing the Speed, Sensitivity and Accuracy of Biomolecular Detection Using Multi-Length-Scale Engineering. Nat. Nanotechnol. 2014, 9, 969-980.

(12) Chang, L.; Rissin, D. M.; Fournier, D. R.; Piech, T.; Patel, P. P.; Wilson, D. H.; Duffy, D. C. Single Molecule Enzyme-Linked Immunosorbent Assays: Theoretical Considerations. J. Immunol. Methods 2012, 378, 102-115.

(13) Tuerk, C.; Gold, L. Systematic Evolution of Ligands by Exponential Enrichment: RNA Ligands to Bacteriophage T4 DNA Polymerase. Science (80-. ). 1990, 249, 505-510.

(14) Ellington, A. D.; Szostak, J. W. In Vitro Selection of RNA Molecules That Bind Specific Ligands. Nature 1990, 246, 818-822.

(15) Robertson, D. L.; Joyce, G. F. Selection in Vitro of an RNA Enzyme That Specifically Cleaves Single-Stranded DNA. Nature 1990, 344, 467-468.

(16) Illiuk, A. B.; Hu, L.; Tao, A. W. Aptamer in Bioanalytical Applications. Anal. Chem. 2011, 83, 4440-4452.

(17) Park, K. S. Nucleic Acid Aptamer-Based Methods for Diagnosis of Infections. Biosens. Bioelectron. 2018, 102, 179-188.

(18) Toh, S. Y.; Citartan, M.; Gopinath, S. C. B.; Tang, T. H. Aptamers as a Replacement for Antibodies in Enzyme-Linked Immunosorbent Assay. Biosens. Bioelectron. 2015, 64, 392-403.

(19) Pei, H.; Zuo, X.; Pan, D.; Shi, J.; Huang, Q.; Fan, C. Scaffolded Biosensors with Designed DNA Nanostructures. NPG Asia Mater. 2013, 5, 1-9.

(20) Cho, E. J.; Lee, J.; Ellington, A. D. Applications of Aptamers as Sensors. Annu. Rev. Anal. Chem. 2009, 2, 241-264.

(21) Chambers, J. P.; Arulanandam, B. P.; Matta, L. L.; Weis, A.; Valdes, J. J. Biosensor Recognition Elements. Curr. Issues Mol. Biol. 2008, 10, 1-12.

(22) Rothemund, P. W. K. Folding DNA to Create Nanoscale Shapes and Patterns. Nature 2006, 440, 297-302.

(23) Liu, J.; Geng, Y.; Pound, E.; Gyawali, S.; Ashton, J. R.; Hickey, J.; Woolley, A. T.; Harb, J. N. Metallization of Branched DNA Origami for Nanoelectronic Circuit Fabrication. ACS Nano 2011, 5, 2240-2247.

(24) Maune, H. T.; Han, S.; Barish, R. D.; Bockrath, M.; Goddard, W. A.; Rothemund, P. W. K.; Winfree, E. Self-Assembly of Carbon Nanotubes into Two-Dimensional Geometries Using DNA Origami Templates. Nat. Nanotechnol. 2009, 5, 61-66.

(25) Yonggang, K.; Stuart, L.; Chang, Y.; Liu, Y.; Yan, H. Self-Assembled Water-Soluble Nucleic Acid Probe Tiles for Label-Free RNA Hybridization Assays. Science (80-. ). 2008, 319, 180-183.

(26) Lin, C.; Liu, Y.; Yan, H. Self-Assembled Combinatorial Encoding Nanoarrays for Multiplexed Biosensing. Nano Lett. 2007, 7, 507-512. 
(27) Godonoga, M.; Lin, T.; Oshima, A.; Sumitomo, K.; Tang, M. S. L.; Cheung, Y.; Kinghorn, A. B.; Dirkzwager, R. M.; Zhou, C.; Kuzuya, A.; et al. A DNA Aptamer Recognising a Malaria Protein Biomarker Can Function as Part of a DNA Origami Assembly. Sci. Rep. 2016, 6, 21266; doi: 10.1038/srep21266.

(28) Vietz, C.; Schu, M. L.; Wei, Q.; Richter, L.; Lalkens, B.; Ozcan, A.; Tinnefeld, P.; Acuna, G. P. Benchmarking Smartphone Fluorescence-Based Microscopy with DNA Origami Nanobeads: Reducing the Gap toward Single-Molecule Sensitivity. ACS Omega 2019, 4 (1), 8-13.

(29) Rothemund, P. W. K.; Papadakis, N.; Winfree, E. Algorithmic Self-Assembly of DNA Sierpinski Triangles. PLOS Biol. 2004, 2, 2041-2053.

(30) Sellner, S.; Kocabey, S.; Nekolla, K.; Krombach, F.; Liedl, T.; Rehberg, M. DNA Nanotubes as Intracellular Delivery Vehicles in Vivo. Biomaterials 2015, 53, 453-463.

(31) Douglas, S. M.; Bachelet, I.; Church, G. M. A Logic-Gated Nanorobot for Targeted Transport of Molecular Payloads. Science (80-. ). 2012, 335, 831-834.

(32) Schuller, V. J.; Heidegger, S.; Sandholzer, N.; Nickels, P. C.; Suhartha, N. A.; Endres, S.; Bourquin, C.; Liedl, T. Cellular Immunostimulation by CpG-Sequence-Coated DNA Origami Structures. ACS Nano 2011, 5 (12), 9696-9702.

(33) Fu, Y.; Zeng, D.; Chao, J.; Jin, Y.; Zhang, Z.; Liu, H.; Li, D.; Ma, H.; Huang, Q.; Gothelf, K. V; et al. Single-Step Rapid Assembly of DNA Origami Nanostructures for Addressable Nanoscale Bioreactors. J. Am. Chem. Soc. 2013, 135, 696-702.

(34) Rinker, S.; Ke, Y.; Liu, Y.; Chhabra, R.; Yan, H. Self-Assembled DNA Nanostructures for Distance Dependent Multivalent Ligand-Protein Binding. Nat. Nanotechnol. 2008, 3 (7), 418-422. https://doi.org/10.1038/nnano.2008.164.Self-assembled.

(35) Douglas, S. M.; Dietz, H.; Liedl, T.; Hogberg, B.; Graf, F.; Shih, W. M. Self-Assembly of DNA into Nanoscale Three-Dimensional Shapes. Nature 2009, 459 (7245), 414-418.

(36) Tikhomirov, G.; Petersen, P.; Qian, L. Fractal Assembly of Micrometre-Scale DNA Origami Arrays with Arbitrary Patterns. Nature 2017, 552, 67-71.

(37) Daems, D.; Pfeifer, W.; Rutten, I.; Sacca, B.; Spasic, D.; Lammertyn, J. Three-Dimensional DNA Origami as Programmable Anchoring Points for Bioreceptors in Fiber Optic Surface Plasmon Resonance Biosensing. ACS Appl. Mater. Interfaces 2018, 10, 23539-23547.

(38) Tran, D. T.; Knez, K.; Janssen, K. P.; Pollet, J.; Spasic, D.; Lammertyn, J. Selection of Aptamers against Ara $\mathrm{H} 1$ Protein for FO-SPR Biosensing of Peanut Allergens in Food Matrices. Biosens. Bioelectron. 2013, 43, 245-251.

(39) Rissin, D. M.; Fournier, D. R.; Piech, T.; Kan, C. W.; Campbell, T. G.; Chang, L.; Rivnak, A. J.; Patel, P. P.; Provuncher, G. K.; Ferrell, E. P.; et al. Simultaneous Detection of Single Molecules and Singulated Ensembles of Molecules Enables Immunoassays with Broad Dynamic Range. Anal. Chem. 2011, 83 (6), 2279-2285.

(40) Huang, Y.; Bell, M. C.; Suni, I. I. Impedance Biosensor for Peanut Protein Ara H 1. Anal. Chem. 2008, 80 (23), 9157-9161.

(41) Schmitt, D. A.; Cheng, H.; Maleki, S. J. Competitive Inhibition ELISA for Quantification of Ara H 1 and Ara H 2, the Major Allergens of Peanuts. J. AOAC Int. 2004, 87 (6), 1492-1497.

(42) Wen, H.-W.; Borejsza-Wysocki; DeCory, T. R.; Durst, R. A. Development of a Competitive Liposome-Based Lateral Flow Assay for the Rapid Detection of the Allergenic Peanut Protein Ara h1. Anal. Bioanal. Chem. 2005, 382, 1217-1226. 
(43) Dunn, K. E.; Dannenberg, F.; Ouldridge, T. E.; Kwiatkowska, M.; Turberfield, A. J.; Bath, J. Guiding the Folding Pathway of DNA Origami. Guid. Fold. Pathw. DNA origami 2015, 525, 8286.

(44) Castro, C. E.; Kilchherr, F.; Kim, D.; Shiao, E. L.; Wauer, T.; Wortmann, P.; Bathe, M.; Dietz, H. A Primer to Scaffolded DNA Origami. Nat. Methods 2011, 8, 221-229.

(45) Stahl, E.; Martin, T. G.; Praetorius, F.; Dietz, H. Facile and Scalable Preparation of Pure and Dense DNA Origami Solutions. Angew. Chemie - Int. Ed. 2014, 53, 12735-12740.

(46) Chruszcz, M.; Maleki, S. J.; Majorek, K. A.; Demas, M.; Bublin, M.; Solberg, R.; Hurlburt, B. K.; Ruan, S.; Mattisohn, C. P.; Breiteneder, H.; et al. Structural and Immunologic Characterization of Ara H 1, a Major Peanut Allergen. J. Biol. Chem. 2011, 286 (45), 39318-39327.

(47) Shaw, A.; Hoffecker, I. T.; Smyrlaki, I.; Rosa, J.; Grevys, A.; Bratlie, D.; Sandlie, I.; Michaelsen, T. E.; Andersen, J. T.; Högberg, B. Binding to Nanopatterned Antigens Is Dominated by the Spatial Tolerance of Antibodies. Nat. Nanotechnol. 2019, 1-11.

(48) Pérez-Ruiz, E.; Decrop, D.; Ven, K.; Tripodi, L.; Leirs, K.; Rosseels, J.; Wouwer, M. Van De; Geukens, N.; De Vos, A.; Vanmechelen, E.; et al. Digital ELISA for the Quantification of Attomolar Concentrations of Alzheimer's Disease Biomarker Protein Tau in Biological Samples. Anal. Chim. Acta 2018, 1015, 74-81.

(49) Pérez-Ruiz, E.; Lammertyn, J.; Spasic, D. Evaluation of Different Strategies for Magnetic Particle Functionalization with DNA Aptamers. N. Biotechnol. 2016, 33 (6), 755-762.

(50) Douglas, S. M.; Marblestone, A. H.; Teerapittayanon, S.; Vazquez, A.; Church, G. M.; Shih, W. M. Rapid Prototyping of 3D DNA-Origami Shapes with caDNAno. Nucleic Acids Res. 2009, 37 (15), 5001-5006. 